\title{
Anorectal Gastrointestinal Stromal Tumour: A Case Treated with Radical Surgery
}

\author{
Arbin Joshi
}

${ }^{1}$ Phect Nepal, Kirtipur Hospital, Kirtipur, Kathmandu, Nepal.

\begin{abstract}
Gastrointestinal Stromal Tumour involving rectum and anal canal is an extremely rare entity. This is a case report of a 47 years lady presented with fresh rectal bleed associated with rectal pain and foul smelling rectal mucus discharge. On rectal examination, she had a firm mass palpable about $1.5 \mathrm{~cm}$ from anal verge. Considering the size of the tumour and its close proximity with cervix and involvement of levator muscles, extralevator abdominal perineal excision of rectum was undertaken with good recovery after surgery. It was followed by imatinib therapy.

Keywords: Anorectum; extra levator abdominal perineal resection; gastrointestinal stromal tumour; imatinib.
\end{abstract}

\section{INTRODUCTION}

Gastrointestinal Stromal Tumour (GIST) is a rare tumour. In western Sweden, annual incidence of clinically detected GIST was estimated 14.5 per million inhabitants as shown by the epidemiological data produced by Nilsson et al. ${ }^{1}$ Stomach is the most frequent site of GIST. ${ }^{2}$ Colon and rectum are the rare site of GIST, whereas GIST involving the anus is extremely rare. ${ }^{1}$ In some series, anorectal GIST accounted for about $5 \%$ of all GIST. ${ }^{3}$

We report a case anorectal GIST, a rare site of presentation of a rare tumour who presented to us in our university level tertiary hospital and was treated with a radical extralevator abdominal perineal excision of rectum (ELAPE).

\section{CASE REPORT}

This is a 47 years lady with history of painless per rectal fresh bleed which was noticed for the first time two years back. For this problem she visited another tertiary centre where she was diagnosed as anorectal GIST about $2 \mathrm{~cm}$ from the anal margin and was confirmed with trucut biopsy and immunohistochemistry (CD 117 positive but $S$ 100 negative). Size of the tumour according to per rectal examination done by the surgeon in that centre was 3 to $4 \mathrm{~cm}$. She was offered local excision in the centre which she denied.

She again presented to our centre with similar per rectal bleed but profuse in amount for last 4 months associated with rectal pain, foul smelling mucus mixed with blood and severe tenesmus. She had insignificant loss of weight and loss of appetite. On examination she was found to be pale and her nutritional status was moderate according to subjective global assessment. Her abdominal examination was unremarkable. On per rectal examination she was found to have firm mass of size of size of $6 \times 8 \mathrm{cms}$, about $1.5 \mathrm{~cm}$ from the anal verg, arising from posterior wall of the rectum extending to the right lateral wall. The mass was tender and immobile. An ulcer could be felt on the surface of the mass and the mass was occluding the lumen of rectum. Blood with dirty mucus was found in the examining finger.

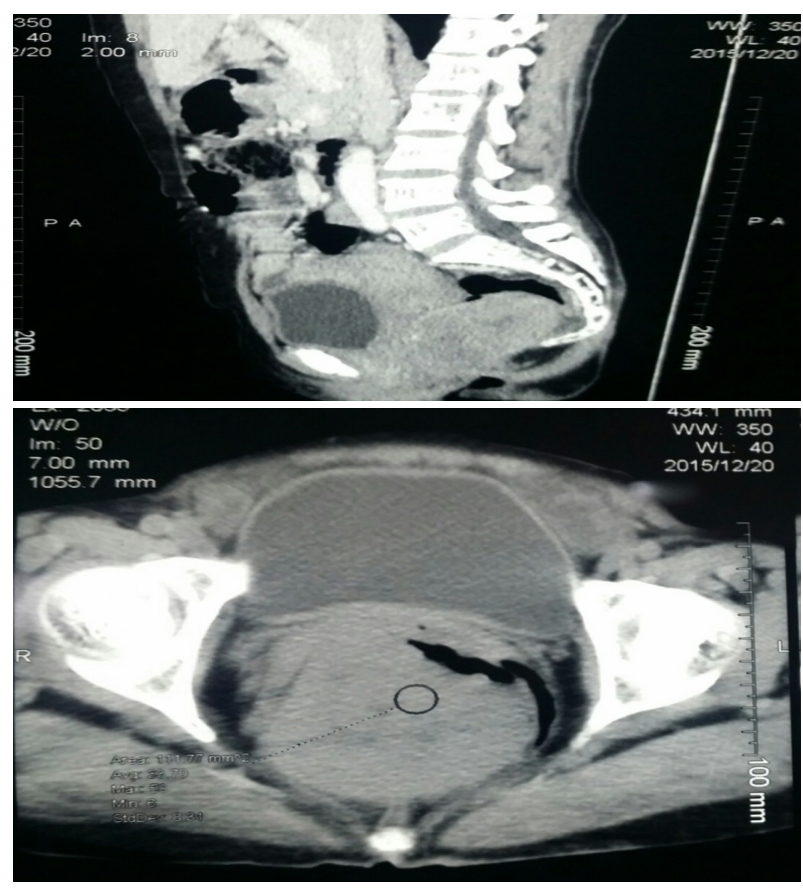

Figure 1. Mass in rectum completely occluding the lumen and compressing the cervix.

Correspondence: Dr Arbin Joshi, Phect Nepal, Kirtipur Hospital, Kirtipur, Kathmandu, Nepal. Email : joshiarbin2012@gmail.com, Phone : 9841286978. 
She was sent for baseline investigations which showed her hemoglobin to be $7 \mathrm{gm} \%$ and her contrast enhanced CT scan of her abdomen and pelvis showed about 3.4 X4.0 X $3.7 \mathrm{~cm}$ moderate heterogenously enhancing mass in anorectal region arising from right lateral wall with perirectal soft tissue strandings and no signs of distant metastasis. There was no bowel distention in the scan. (Fig 1)

Considering the size of the tumour and its close proximity with cervix and involvement of levator muscles, extralevator abdominal perineal excision of rectum was planned. Patient was brought to operating room and under general anaesthaesia exploratory laparotomy was done and rectosigmoid mobilisation was done along the mesorectal plane. A gauze piece was left in the presacral space. Proximal sigmoid was divided and after closing the distal end, proximal end was brought out of pre marked site at left iliac fossa for permanent colostomy. Abdomen was closed, stoma created and patient was turned to prone jack-knife position. With a tear drop incision coccyx was incorporated with the specimen. Anteriorly, part of vaginal wall was also incorporated with the specimen and extralevator resection of rectum was completed. The resulting defect (Fig 2) was closed primarily with number 1 polypropylene in two layers and skin was left open.

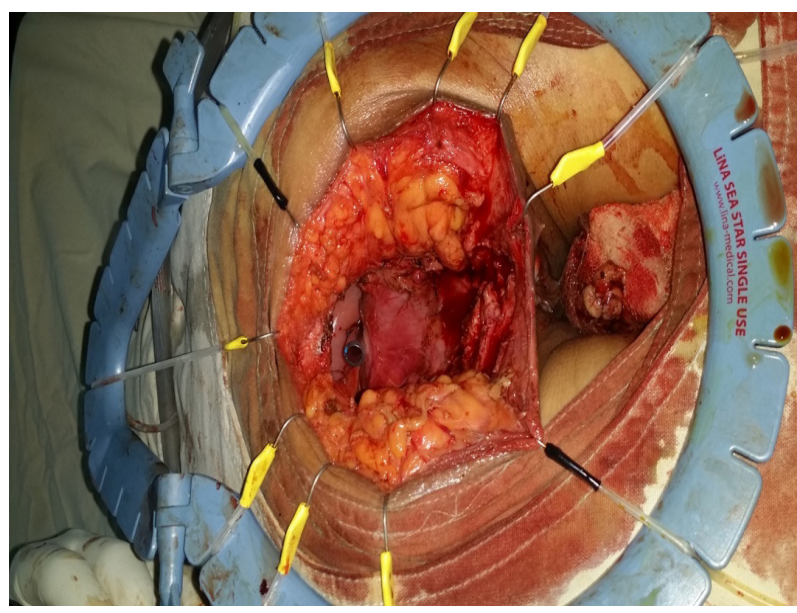

Figure 2. Defect in the perianal region after removal of the specimen.

Patient recovered well and was discharged on day 5 . Perineal wound was healed completely on day 10 follow up. Histopathological result showed GIST of maximum tumor dimension $6.2 \mathrm{cms}$ with free resected margins and with 8 mitotic figure per 50 High Power Field (HPF); pathological staging being T3 NO Mx. She has been started on adjuvant imatinib therapy and a regular follow up planned.

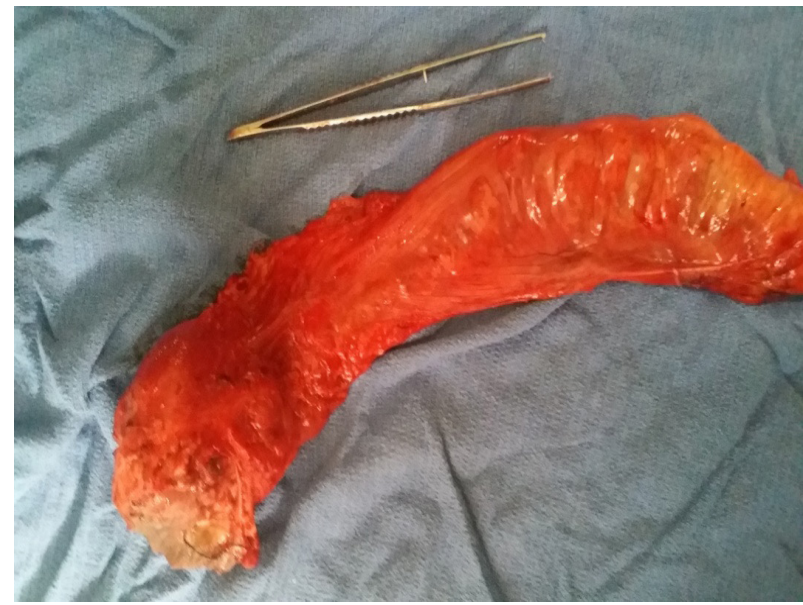

Figure 3. Cylindrical Specimen of rectum with anal canal and sigmoid colon removed.

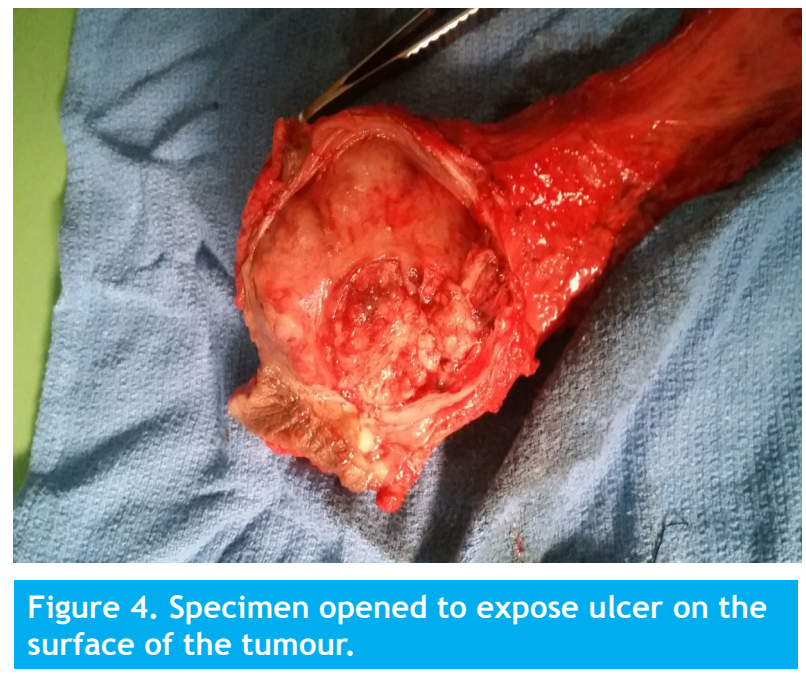

\section{DISCUSSION}

Anorectal GIST make up only $0.1 \%$ of all tumours originating in the rectum, underlining the rarity of this condition. ${ }^{4}$ Per rectal bleed and rectal pain is the commonest mode of presentation. However, large GIST in anal canal can present as urinary retention. ${ }^{5}$

Surgical treatment for GIST at this rare location is controversial. We opted for radical ELAPE taking into consideration the size of the tumour, involvement of the anal canal and failure to identify clear demarcation of the tumour with cervix. We succeeded in achieving RO resection this way which would not have been possible with local excision in this size of the tumour. The final histology labeled this tumour as high risk which again justified our approach.

There are studies that show less extensive excision achieving R0 resection. One such case report was from Nigri et $\mathrm{al}^{2}$ showed successful local excision sparing the anal sphincters for the tumour of size 3.5 
$\mathrm{cm}$. Another case report from Kumar et $\mathrm{al}^{6}$ undertook abdominoperineal resection (APR) for tumour of size 2 $X 1.5 \mathrm{~cm}$. These case reports emphasized that there were only eleven cases of c-kit positive anal GIST are reported in literature so far which is not sufficient to establish a widely accepted approach. However, another case reported $14 \mathrm{~cm}$ sized anorectal GIST needed APR. ${ }^{5}$ Hence, controversy of whether to go for radical approach or not is still on.

Radical treatment with APR might be needed if the size of the tumour is large for RO resection and to avoid local recurrence. Radical surgery is recommended for anorectal tumour of size of $>5 \mathrm{~cm} .{ }^{7,8}$ Vorobyov et al. ${ }^{9}$ in the series of 36 rectal GISTs reported no recurrence in tumour size ranging from 8 to $20 \mathrm{~cm}$ who underwent abdominoperineal resection but of the remaining patients who underwent transanal, pararectal or transvaginal excision had recurrence and malignant transformation. This clearly shows high recurrence and malignant transformation in patients undergoing local excision by any route. However, literature has shown there is no significant difference in distant metastasis or survival in between APR or conservative surgery, adding further confusion to the issue. ${ }^{10}$

In post-imatinib era, we can presume less local recurrence after radical R0 surgery. A randomized controlled trial in this subject may not be possible because of the rarity of this condition and could be unethical. Imatinib as neoadjuvant therapy is another gray area. Though no proper guidelines have come up yet, there are some reports that shows good response of imatinib in anorectal GIST regarding tumour shrinkage, allowing surgeons to preserve anal sphincters rather than going for APR and permanent colostomy. ${ }^{11}$ NCCN recommends treatment with imatinib for those who have potentially resectable disease but with the risk of significant morbidity. Neoadjuvant imatinib for identifiable high risk characteristics such as size $>5 \mathrm{~cm},>5$ mitoses per 50 $\mathrm{HPF}$, anatomic location resulting in a potentially morbid resection seems like a reasonable approach. ${ }^{12}$ Probably less radical surgery could be the option in the future once efficacy of imatinib as neoadjuvant therapy will be well established in case of anorectal GIST.

\section{CONCLUSIONS}

Hence, radical excision for anorectal GIST of size more than $5 \mathrm{~cm}$ followed by imatinib therapy should be the recommended practice until efficacy of neoadjuvant imatinib for this condition is well established.

\section{REFERENCES}

1. Nilsson B, Bümming $\mathrm{P}$, Meis-Kindblom JM, Odén A, Dortok A, Gustavsson B, Sablinska K, Kindblom LG. Gastrointestinal stromal tumors: the incidence, prevalence, clinical course, and prognostication in the preimatinib mesylate era. Cancer. 2005;103(4):821-9.[Full Text]

2. Nigri GR, Dente M, Valabrega S, Aurello P, D'Angelo F, Montrone G, Ercolani G, Ramacciato G. Gastrointestinal stromal tumor of the anal canal: an unusual presentation. World J Surg Oncol. 2007;5(1):20.[Bio Med]

3. Miettinen M, Sarlomo-Rikala M, Lasota J: Gastrointestinal stromal tumours. Ann Chir Gynaecol. 1998;87:278-281.

4. Tran T, Davila JA, El-Serag HB. The epidemiology of malignant gastrointestinal stromal tumors: an analysis of 1,458 cases from 1992 to 2000. Am J Gastroenterol. 2005;100(1):162-8.[Full Text]

5. Mehta G, Verma S, Inamdar P. Anorectal Leiomyoma: Case Report of Rare Tumor with unusual Presentation. Indian J Surg Oncol. 2010;1(4):337-40.[Full Text]

6. Kumar M, Goel MM, Singh D. Rare case of gastrointestinal stromal tumor of the anal canal. J Cancer Res Ther. 2013;9(4):736..[Full Text]

7. Le Borgne J, Guiberteau-Canfrere V, Lehur PA, Bitar O, Serraz H, Thoulouzan E, Le Bodic MF. Leiomyoma of the rectum. Chirurgie. 1993;119(4):212-5.[Full Text]

8. Nemer FD, Stoeckinger JM, Evans OT. Smooth-muscle rectal tumors: a therapeutic dilemma. Dis Colon Rectum. 1977;20(5):405-13.[Full Text]

9. Vorobyov GI, Odaryuk TS, Kapuller LL, Shelygin YA, Kornyak BS. Surgical treatment of benign, myomatous rectal tumors. Dis Colon Rectum. 1992;35(4):328-31. [Full Text]

10. Changchien CR, Wu MC, TasiWS, Tang R, Chiang JM, Chen JS, Huang SF, Wang JY, Yeh CY. Evaluation of prognosis for malignant rectal gastrointestinal stromal tumor by clinical parameters and immunohistochemical staining. Dis Colon Rectum. 2004;47(11):1922-9.[Full Text]

11. Wang JP, Wang T, Huang MJ, Wang L, Kang L, Wu XJ. The role of neoadjuvant imatinib mesylate therapy in sphincterpreserving procedures for anorectal gastrointestinal stromal tumor. Am J Clin Oncol. 2011;34(3):314-6.[Full Text]

12. von Mehren M, Watson JC. Perioperative tyrosine kinase inhibitors for GIST: standard... or an idea that needs further investigation?. Oncology (Williston Park, NY). 2009;23(1):65. [Full Text] 